\title{
Introduction: Autonomy in Healthcare
}

\author{
James Stacey Taylor ${ }^{1}$
}

Published online: 10 August 2018

(c) Springer Nature B.V. 2018

Autonomy is one of the central concepts in medical ethics. Indeed, so dominant has it become that of the four principles of biomedical ethics that Beauchamp and Childress outline in their seminal Principles of Biomedical Ethics (respect for autonomy, beneficence, nonmaleficence, and justice) respect for autonomy can rightly be said to be the "first among equals". ${ }^{1}$ Unfortunately, despite the dominance of autonomy in medical ethics, the development of a theoretically satisfying account of autonomy that can play the role that it is often called to play in the practice of medicine has been challenging. The conception of autonomy that is often drawn upon in bioethical discussions is often much thinner than that which is developed by action theorists who are concerned with identifying the conditions that must be met for a person to be autonomous with respect to (variously) her desires, decisions, or actions. Indeed, bioethicists often ascribe autonomy to a person with respect to, for example, her acts merely if she understood what she was doing when she performed them and was free from undue influence. This approach might be appropriate in the initiation of bioethical discussion of a particular clinical case-and it might even lead to a resolution that all parties could agree to. However, it might also be the case that the thinness of this account of autonomy could preclude the making of any practical decisions even among persons who agree that autonomy is the primary value to be used to decide the case at hand. This is because the very thinness of this approach that was supposed to render the concept of autonomy sufficiently graspable to be useful in practical decision-making might be self-defeating, undermining its usefulness as the discussion reveals that it is insufficiently robust to provide concrete guidance. One might, for example, question just how much a person needs to understand about her situation and the effects of her proposed courses of action upon it in order to count as autonomous with respect to her decisions. Or, one might question what counts as being free from "undue" influence. When such difficulties arise, it

\footnotetext{
1 Beauchamp and Childress (2012). The phrase "first among equals" was used in this context by Gillon (2003).
}

James Stacey Taylor

jtaylor@tcnj.edu

1 Department of Philosophy, Religion, and Classical Studies, The College of New Jersey, Ewing, USA 
becomes clear that no matter how far removed the practical discussions of what to do in a particular medical situation to respect the value of autonomy might appear to be from the theoretical analysis of this concept, they need a robust theoretical grounding so that questions such as this can be answered when they occur.

Lucie White, recognizing the need for a robust theoretical grounding of the concept of autonomy so that it can play the role that it is intended to play in practical bioethical discussion, argues in the first paper in this Special Issue of Healthcare Ethics Committee Forum that the theoretical focus on "authenticity" (the relationship that a person's desire must have to her self for her to be autonomous with respect to it) should inform accounts of autonomy that inform discussions in medical practice. In her paper, "The Need for Authenticity-Based Autonomy in Medical Ethics", White argues that it is only through drawing on the concept of authenticity that we can effectively address concerns that might arise in a medical context as to whether or not a person's desires are truly her own (e.g., when a person's medical decisions are highly unusual) and thus should be accorded the respect that is due to a desire with respect to which a person is autonomous.

A concern to combine the theoretical and practical discussions of autonomy also motivates Steven Weimer's discussion in his paper, "Evidence Responsiveness and the Ongoing Autonomy of Treatment Preferences". Noting that autonomous agents direct their own lives, Weimer observes that this would require that they exercise ongoing control over the path they wish to pursue. Given this, he argues, a patient who was able autonomously to consent to her treatment but who becomes unable to continue to assess whether or not she could continue with it will lack autonomy with respect to her ongoing treatment. But, notes Weimer, this feature of autonomy has received very little theoretical attention. To rectify this circumstance, Weimer provides a way to account for this temporal aspect of autonomy that is not only theoretically satisfying but which can be drawn upon in the practical debates of medical ethics.

Yet, not everyone agrees that a theoretically-rich account of autonomy is needed to undergird the practical discussions of medical ethics where this concept has recently come to play a prominent role. Joseph T. F. Roberts, in his contribution to this Special Issue, argues in his paper, "Autonomy, Competence, Non-interference", that the concept of competence is better placed than that of autonomy to play the antipaternalist role that the latter concept often plays in contemporary medical ethics. For Roberts, individuals should be granted rights against interference by others once it has been established that they are competent; that they can acquire knowledge, exercise instrumental rationality, and form a life-plan which they can revise as they see fit. Roberts argues that this account of competence captures the contours of the concept, which, in turn, is less contested than that of autonomy. Since this is so, we can infer from Roberts' initial discussion of the varieties of autonomy theories, an emphasis on competence rather than on autonomy will enable us to make progress in our practical discussions without having first to reach a consensus about the conditions that must be met for a person to be autonomous with respect to, for example, her decisions.

John K. Davis also takes up the question of what counts as competence in his paper, "Dr. Google and Premature Consent". Davis addresses the problem of patients who have made up their minds about a diagnosis or a treatment plan prior to meeting with their healthcare provider, having "researched" their medical condition on their own. 
He argues that these patients are not truly competent-and so their decisions should not provide the bulwark against interference that Roberts argues competent decisions should be held to-for they are not deliberating well when they make medical decisions prior to seeing a healthcare professional. Moreover, he argues, such patients are also not truly informed, for they are unable accurately to determine which sources of medical information they should trust, and they might fail to believe information that they are provided with.

The question of the nature and importance of informed consent that Davis raises is taken up by Paul Hamilton in the fifth paper in this Special Issue, "A Republican Argument Against Nudging and Informed Consent". Hamilton argues that it would violate a person's informed consent to her treatment if she had been guided into it by the use of a "nudge". Hamilton argues that such nudges places physicians in a position of dominance over their patients. This, argues Hamilton, violates the republican freedom of the patients insofar as they are no longer free from the possibility of arbitrary interference by another - a position that has clear affinities with that of Roberts earlier in this Special Issue. As a way of avoiding nudging, Hamilton suggests that patients be paired with physicians who share their values.

Davis' and Hamilton's concerns about the type of information that patients have access to and base their decisions upon is shared by Thomas May and Harold Grotevant. May and Grotevant argue that a concern for patient well-being supports providing patients with their genetic-relative family health history in their paper, "Autonomy, Well-being, and the Value of Genetic Testing for Adopted Persons". This Special Issue then concludes with a discussion by Maura Priest in her paper, "Autonomy Centered Healthcare", of the effects that the current approach to health insurance that is taken by the United States has upon the autonomy of both physicians and patients.

As this selection of papers indicates, contemporary discussions of autonomy in the context of healthcare are rich and varied. This selection of papers also shows that such discussions can be helpful in illuminating questions in medical ethics that arise as a result of new technology, either those that arise directly from it (such as, those generated by advances in genetic testing) or those that are generated more indirectly (such as, those engendered by patients' access to vast amounts of information through the Internet). And, of course, this selection reinforces the long-held view in political theory that a concern for the value of autonomy should be used to assess the merits of policy proposals, such as, for example, nudging or new methods of providing health insurance. It is thus hoped that this Special Issue will contribute to both the theoretical understanding of autonomy and to those debates in which it plays a role, and in which the contributors to this Special Issue have thereby engaged with.

\section{References}

Beauchamp, T. L., \& Childress, J. F. (2012). Principles of biomedical ethics. Oxford: Oxford University Press.

Gillon, R. (2003). Ethics needs principles-Four can encompass the rest-And respect for autonomy should be 'first among equals'. Journal of Medical Ethics, 29(5), 307-312. 